\title{
Ability of a Robot to Travel Through its free Work Space in an Environment with Obstacles
}

\author{
Philippe Wenger, Patrick Chedmail
}

\author{
Laboratoire d'Automatique de Nantes \\ Ecole Nationale Supérieure de Mécanique de Nantes, France
}

\begin{abstract}
This article presents a new geometric analysis of the free work space of a robot among obstacles. The free work space $(F W)$ is defined as the set of positions and orientations that the robot's end effector can reach, according to the joint limits and the various obstacles lying in the environment. The aim is to give global descriptions of the robot's ability to move in the operational space (which coincides with Cartesian space when only position coordinates are specified).

The main contribution of this work is the characterization of the effects of obstacles on the work space geometry, as well as on its topology. The ability of a robot to move freely in its work space (called the "moveability") is difficult to describe and needs stringent formalizations. The concept of moveability is introduced through various properties and their corresponding necessary and sufficient conditions. Using a Constructive Solid Geometry (CSG) Computer-Aided Design (CAD) description of robots and obstacles and an octree model of the $F W$, these properties permit characterization of selected moveability areas in the $F W$, where, for instance, any $\mathrm{n}$ points can be linked together or where any continuous trajectory can be achieved without changing configuration. This new global description is of great interest for the user of CAD systems when designing robotic cells.
\end{abstract}

\section{Introduction}

Automatic Design or Computer Aided Design of robotic cells is actually an important challenge in industry and involves several difficult geometric problems:

- the choice of a robot; the difficulty is to find the morphology (type and number of joints, length of links, ... .) that is best suited to the family of tasks to be achieved.

- the geometric layout of the cell; one has to position the robot(s) and the other components of the cell in such a way that the robot is able to work conveniently in the environment (accessibility of the specified work areas, as well as mobility in them, should be ensured).

- Collision-free paths planning; the problem is to find a continuous path among obstacles between one specified location to another.

This third problem has drawn the interest of many authors, and its theoretical solution is now well known (Schwartz and Sharir 1982; Brady et al. 1982; Canny and Reif 1987). The techniques are various: for instance, the local approaches with potential methods (Khatib 1985; Koditschek 1987) or the global ones based on the configuration space analysis and using cell decomposition (Faverjon 1984; Brooks 1983a,b; Lozano-Pérez 1986) or retraction techniques such as stratified sets (Canny 1987) and Voronoi diagrams (O'Dunlaing et al. 1984; Canny and Donald 1988). These studies have already given rise to operational systems (Lozano-Pérez et al. 1987).

On the other hand, the first two problems have not been so extensively studied. Softwares of current robotics computer-aided design (CAD) systems (such as ROBCAD from Teknomatix, MACAUTO from McDonnel-Douglas, CATIA from Dassault System, or ROBOT PLUS from Computervision) all require the action of a human operator who selects the right solution, validates the choices, and modifies the parameters if necessary (Bernard 1984; Dombre et al. 1986; Deligneres 1987). This article deals with these first two problems. For both of them, characterization and analysis of the free work space are well-suited aids. The free work space (FW) of a robot among obstacles 
is the space of positions and orientations of the end effector according to the joint limits and the various obstacles. Concerning the first problem, criteria such as volume, connectedness, compactness of the work space, or dexterity in it are very convenient for the optimization of the robot's morphology, as they give a good evaluation of the geometric performances of the manipulator (Vijaykumar et al. 1986; Lenarcic et al. 1988). A possible solution to the second problem is the characterization of selected regions of "moveability" in the work space. The aim is then to ensure the feasibility of tasks defined as areas to be freely reached (Deligneres 1987) and/or to be "travelled through" in a certain sense by the robot (e.g., areas of continuous welding or painting). In Figure 1, the piece cannot be placed anywhere in the free work space; it must be enclosed in a region reachable from configurations $\mathbf{q}_{2}>0$ or $\mathbf{q}_{2}<0$ separately.

The goal of our study is to give a global description of the obstacles in the operational space (space of positions and orientations of the end effector; this space coincides with the Cartesian space when only position coordinates are specified). This is achieved through geometric and topologic analysis of the FW. This new description is of great interest for the designer of robotic cells.

A lot of work has already dealt with work space analysis. Typical geometric characterizations of a work space are:

1. Its well-known projections in the Cartesian space: "reachable work space" (projection of the whole

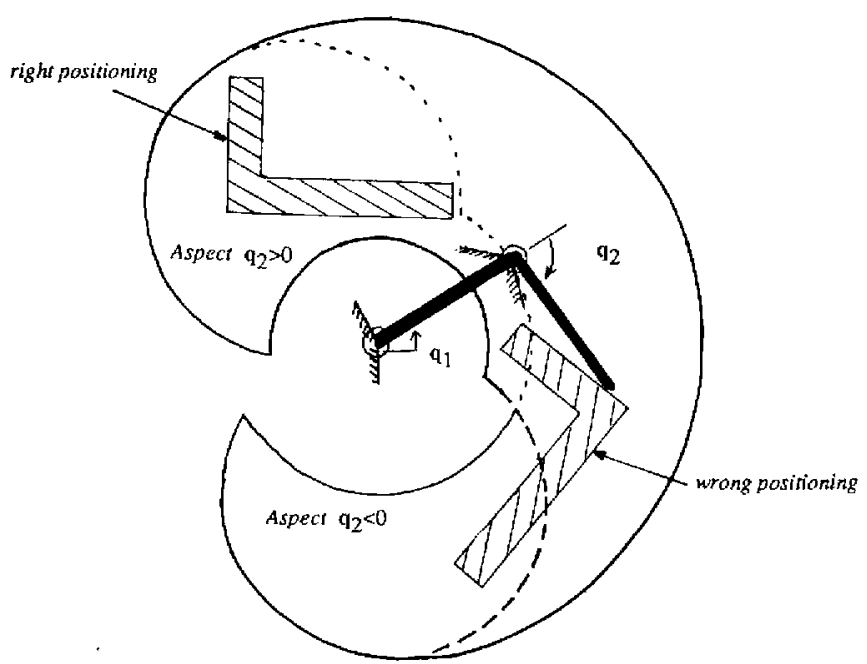

Fig. I. The task to be achieved is continuous welding of a piece. The piece must be carefully placed in the work space to avoid configuration changing during the operation. The end effector must remain in the same aspect. work space) and "dextrous work space" (projection of the work space part where every orientation of the end effector is possible) (Kumar and Waldron 1981).

2. The analysis of holes and voids in the work space (Gupta and Roth 1982).

3. The approach angles and lengths (Hansen et al. 1983).

4. Work area analysis (Yang and Chiueh 1986).

5. Characterization of the so-called "aspects" (set of positions and/or orientations reachable under given configurations) (Borrel 1986).

6. "Well-connectedness" (ability to move between any two points in the work space without changing configuration) (Paden and Sastry 1988).

However, none of these take into account the effects of obstacles. The proximity of obstacles not only modifies the shape of the work space, but also reduces the moveability of the robot within it, as has already been shown in Chedmail and Wenger $(1987 ; 1988)$. Figure $2 A$ shows a very simple example where the FW is connected but does not allow the robot to travel through it. In Figure $2 B$, the mobility between any two points within the FW is ensured, but not necessarily along a given continuous trajectory.

In this article, we propose five stringent characterizations of the moveability in the FW for a robot among obstacles. We attach five necessary and sufficient conditions to these characterizations. These conditions are tested using a constructive solid geometry (CSG) CAD technique (Martin et al. 1985) and an octree model of the FW. Finally, an algorithm is described to check the various properties, and results are presented.

\section{Definitions}

We give in this section some definitions before proceeding further.

Let a robot-called ROBOT(q) - be defined by its $n$ degrees of freedom:

$R O B O T(q)$, enclosed in $\mathbb{R}^{3}$, is defined as a set of $n$ solids, as in Wenger (1985) and Yu and Khalil (1986).

Let an environment with obstacles be defined by

$O b=$ set of the physical obstacles of the environment, enclosed in $\mathbb{R}^{3}$

Let $f: \mathbb{R}^{n} \rightarrow \mathbb{R}^{m}$ be the geometric operator of the robot (Khalil and Kleinfinger 1986), where $m$ is the number of operational coordinates of the end effector of the robot, $m \leqslant 6$. 

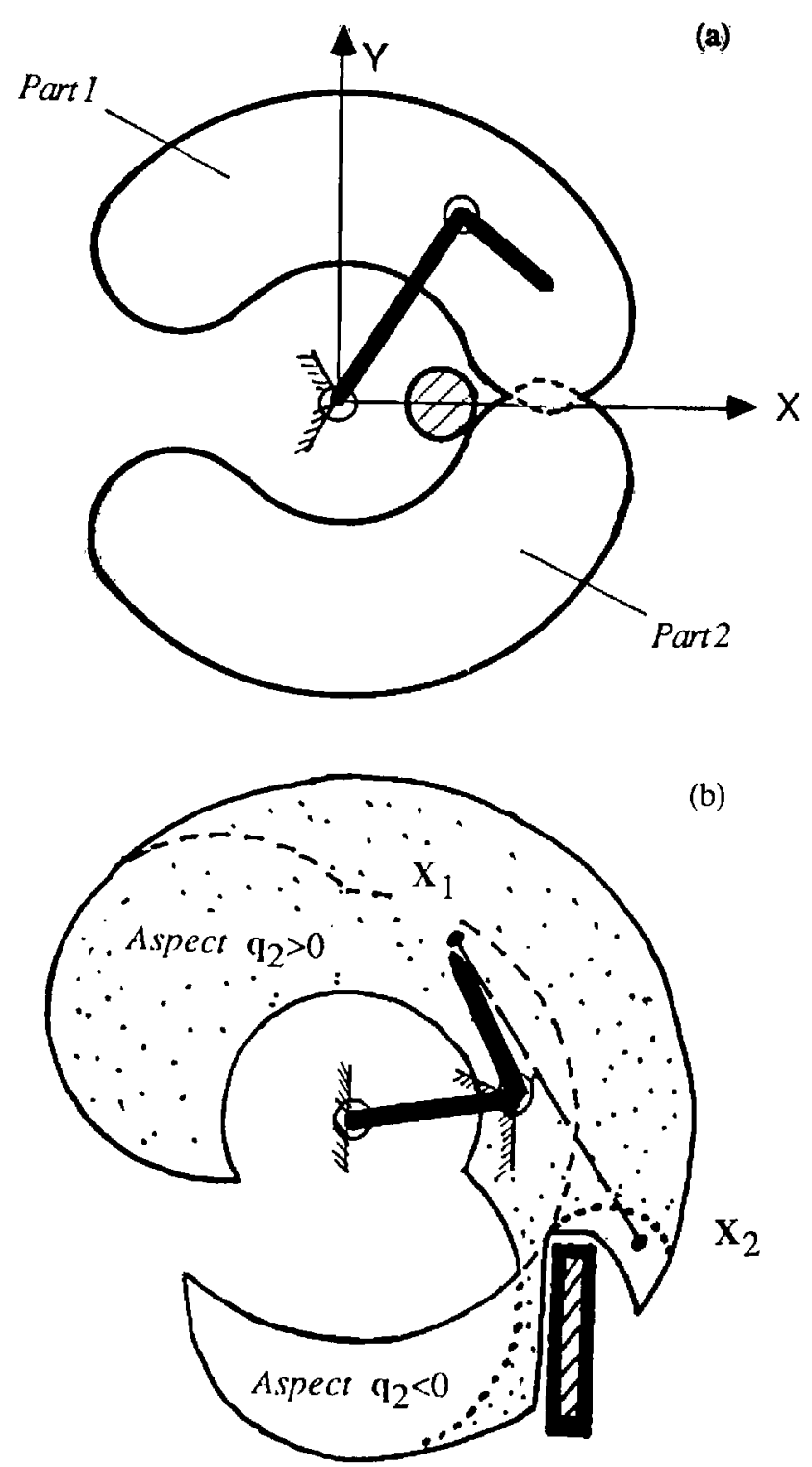

Fig. 2A, A two-DOF plannar linkage with one circular obstacle and $-120^{\circ} \leqslant \mathrm{q}_{1} \leqslant 120^{\circ},-180^{\circ} \leqslant \mathrm{q}_{2} \leqslant 180^{\circ}$. The free work space is connected; however, the robot cannot travel through it but can only move separately within part 1 or part $2 \mathrm{~B}, A$ two-DOF plannar linkage with one rectangular obstacle and $-90^{\circ} \leqslant \mathrm{q}_{1} \leqslant 90^{\circ},-100^{\circ} \leqslant \mathrm{q}_{2} \leqslant 120^{\circ}$. The robot can join any two points in its free work space but not along any trajectory (the given continuous trajectory is unfeasible; point $X_{1}$ can be reached only in aspect 1 because of the joint limits, and point $X_{2}$ can be reached only in aspect 2 because of the obstacle).

Let $\mathscr{D}=\left\{\mathbf{q} \in \mathbb{R}^{n} / \forall i, q_{\text {imax }} \leqslant q_{i} \leqslant q_{\text {imin }}\right.$ ) be the configuration space, according to the joint limits.

Let $Q f$ be the configuration collision-free space of $R O B O T(\mathbf{q})$ :

$$
Q f=\{\mathbf{q} \in \mathscr{D} / \operatorname{ROBOT}(\mathbf{q}) \cap O b=\varnothing)
$$

Let $W f$ be the FW of $R O B O T(q)$. It is the image of $Q f$ under the geometric operator:

$$
W f=f(Q f)
$$

The free work space is the space of positions and orientations of the end effector according to the joint limits and the various obstacles in the environment. A point $\mathbf{X}$ in $W f$ is a vector of $m$ operational coordinates.

We suppose that there are $N$ connected components of $Q f$. We shall denote them as:

$$
\left\{Q f_{i}: i \in I\right\} \text { where } I \text { is the set }\{1, \ldots, \mathrm{N}\} \text {. }
$$

The following relations hold:

$$
Q f=\cup_{i \in I}\left(Q f_{i}\right)
$$

and $\forall i \neq j, i \in I, j \in I ; Q f_{i} \cap Q f_{j}=\varnothing$; and $Q f_{i} \cup Q f_{j}$ is not connected.

Then:

$\forall i \in I: W f_{i}=f\left(Q f_{i}\right)$ is the image of $Q f_{i}$ under the geometric operator.

The following relation holds:

$$
W f=\cup_{i \in I} W f_{i}
$$

Define: The aspects are defined as in Borrel (1986); an aspect is a subspace $A$ enclosed in $Q f$ such that:

1. $A$ is connected,

2. $\forall \mathbf{q} \in A$; the determinant of any $m \times m$ matrix extracted from the Jacobian matrix $\mathbf{J}$ is not equal to zero, except if this minor is equal to zero everywhere in $\mathscr{D}$. The components of $\mathbf{J}$ are:

$$
J_{i j}=\frac{\partial x_{i}}{\partial q_{j}}
$$

where $q_{j}$ is the $\mathrm{j}$ th component of $\mathbf{q}$, and $x_{i}$ is the ith operational coordinate of $\mathbf{X}=f(\mathbf{q})(1 \leqslant i \leqslant m$ and $1 \leqslant j \leqslant n$.).

The number of aspects is finite. $\left\{A_{j}\right\}_{j \in J}$ is a partition of $\mathscr{D}$.

\section{Characterization of the Robot's Ability to Move Through $\boldsymbol{W f}$}

We expose in this section the five characterizations of the moveability in the FW of a robot, and their corresponding necessary and sufficient conditions.

\subsection{First Characterization and Corresponding Necessary and Sufficient Condition}

The FW can be travelled through by the robot in the sense of $\mathrm{P}_{1}$ if, by definition, any two points in $W f$ can 
be joined by the end effector. This means that for any two points in the FW, a connected component of the configuration collision-free space exists such that its image under $f$ contains both of these points:

$$
\forall \mathbf{X}_{1}, \mathbf{X}_{2} \in W f, \exists i \in I, \mathbf{x}_{1} \in W f_{i} \text { and } \mathbf{X}_{2} \in W f_{i}
$$

Note: This first property can be expressed in a more succinct way:

$$
\left(\mathbf{P}_{1}\right) \Leftrightarrow W f^{2}=\cup_{i \in I} W f_{i}^{2}
$$

$W f$ satisfies $\mathrm{P}_{1}$ if and only if $W f$ is connected, and there exists a family $\left\{I_{1}, I_{2}, \ldots, I_{p}\right\}=\left\{I_{k} / k \in K\right\}$ of subsets of $I$ such that:

$$
\begin{array}{ll}
\text { i. } & \forall k \in K: W f=\cup_{j \in I k} W f_{j} \\
\text { ii. } & W f=\cup_{k \in K}\left(\cap_{j \in I k} W f_{j}\right)
\end{array}
$$

(See the demonstration in Appendix A.)

Example: If $Q f$ is composed of three connected components, the following relations hold:

$$
\begin{aligned}
I & =\{1,2,3\} \\
Q f & =Q f_{1} \cup Q f_{2} \cup Q f_{3} \\
W f & =W f_{1} \cup W f_{2} \cup W f_{3}
\end{aligned}
$$

Let $\left\{I_{k} / k \in K\right\}=\left\{I_{1}, I_{2}, I_{3}\right\}$ with:

$$
I_{1}=\{1,2\}, I_{2}=\{2,3\}, I_{3}=\{1,3\} .
$$

Assume:

$$
W f=W f_{1} \cup W f_{2}, W f=W f_{2} \cup W f_{3}, W f=W f_{1} \cup W f_{3}
$$

and

$$
W f=\left(W f_{1} \cap W f_{2}\right) \cup\left(W f_{2} \cap W f_{3}\right) \cup\left(W f_{1} \cap W f_{3}\right)
$$

Then $W f$ satisfies $\mathrm{P}_{1}$ (Fig. 3).

COROLLARY $1 \quad W f_{p} \subset W f$ satisfies the generalized property $\mathrm{P}_{1}(W f p)$ where:

$$
\begin{aligned}
\left(\mathbf{P}_{1}(W f p)\right) & \forall\left(\mathbf{X}_{1}, \mathbf{X}_{2}\right) \in W f p^{2}, \exists i \in I, \mathbf{X}_{1} \in W f_{i} \cap W f p \\
& \text { and } \mathbf{X}_{2} \in W f_{i} \cap W f p
\end{aligned}
$$

if and only if there exists a family $\left\{I_{1}, I_{2}, \ldots, I_{p}\right\}=$ $\left\{I_{k} / k \in K\right\}$ of subsets of $I$ such that:

$$
\begin{aligned}
\text { i. } & W f p \subset \cap_{k \in K_{K}}\left(\cup_{j \in I k} W f_{j}\right) \\
\text { ii. } & W f p \subset \cup_{k \in K}\left(\cap_{j \in I k} W f_{j}\right)
\end{aligned}
$$

which can be written in the following more succinct way:

$$
W f p \subset\left(\cap_{k \in k}\left(\cup_{j \in I k} W f_{j}\right)\right) \cap\left(\cap_{k \in K}\left(\cap_{j \in I k} W f_{j}\right)\right)
$$

Note: Because any subset of set satisfying $\mathrm{P}_{1}(W f p)$ also satisfies $\mathbf{P}_{1}$ ( $W f p$ ), such subsets are not necessarily connected.
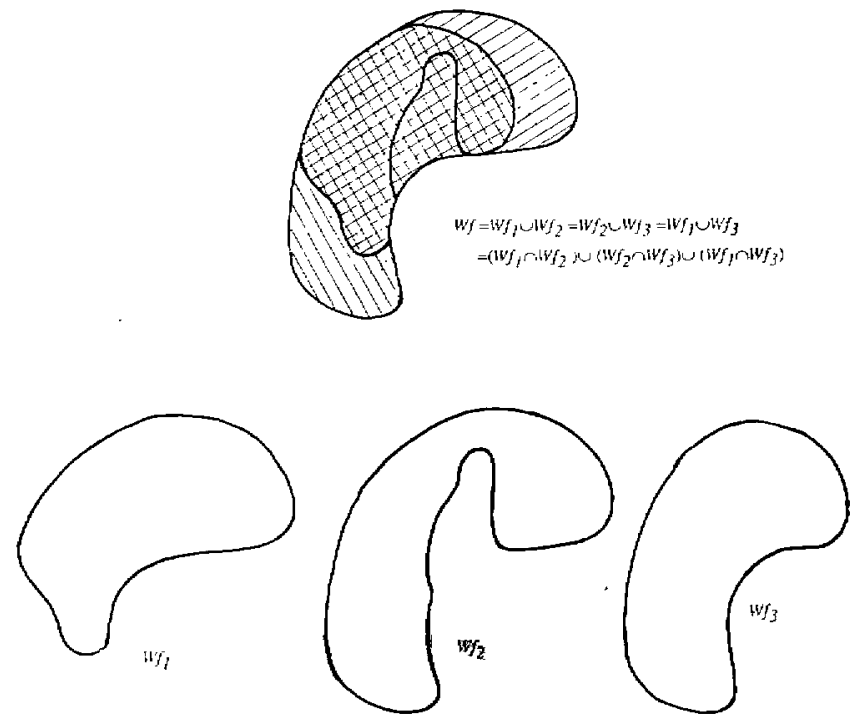

Fig. 3. A case where the free work space can be traveled through in the sense of $P_{1}$.

\section{Definition 1}

The maximal parts $W f p$ of $W f$ satisfying $\mathrm{P}_{1}(W f p)$ are defined as follows:

There exists a family $\left\{I_{1}, I_{2}, \ldots, I_{p}\right\}=\left\{I_{k} / k \in K\right\}$ of subsets of $I$ such that:

$$
W f p=\left(\cap_{k \in \mathcal{K}}\left(\cup_{j \in I k} W f_{j}\right)\right) \cap\left(\cup_{k \in \mathcal{K}}\left(\cap_{j \in I k} W f_{j}\right)\right) \text { is nonempty. }
$$

Note 1: Any part satisfying $\mathrm{P}_{1}(W f p)$ is enclosed in such a maximal part. The converse being true, it is interesting to find the maximal parts satisfying $P_{1}$ (see Fig. $4 A$; in the case where $\operatorname{Card}(I)=3$, there are four maximal parts satisfying $\mathrm{P}_{1}(W f p)$ ).

Note 2: The properties $\mathrm{P}_{1}$ and $\mathrm{P}_{1}(W f p)$ do not take into account initial and final configurations. Moreover, every trajectory in the FW between $\mathbf{X}_{1}$ and $\mathbf{X}_{2}$ is not necessarily achievable. That is why we will complete this definition in the following property; then, in the next two properties, we will successively take into account the configuration at point $\mathbf{X}_{1}$ (or $\mathbf{X}_{2}$ ) and then at points $\mathbf{X}_{1}$ and $\mathbf{X}_{2}$; finally, we will propose a property of mobility along any continuous trajectory through the FW.

\subsection{Second Definition $\left(P_{2}\right)$ and Corresponding Necessary and Sufficient Condition}

The FW can be traveled through by the robot in the sense of $\mathrm{P}_{2}$ if, by definition, every discrete trajectory $\mathbf{T}_{d}$ in $W f$ is achievable, which means:

$\left(\mathrm{P}_{2}\right) \quad \forall \mathbf{T}_{\mathbf{d}}$ discrete trajectory in $W f, \exists i \in I$ such that $\mathbf{T}_{\mathbb{d}} \subset W f_{i}$ 

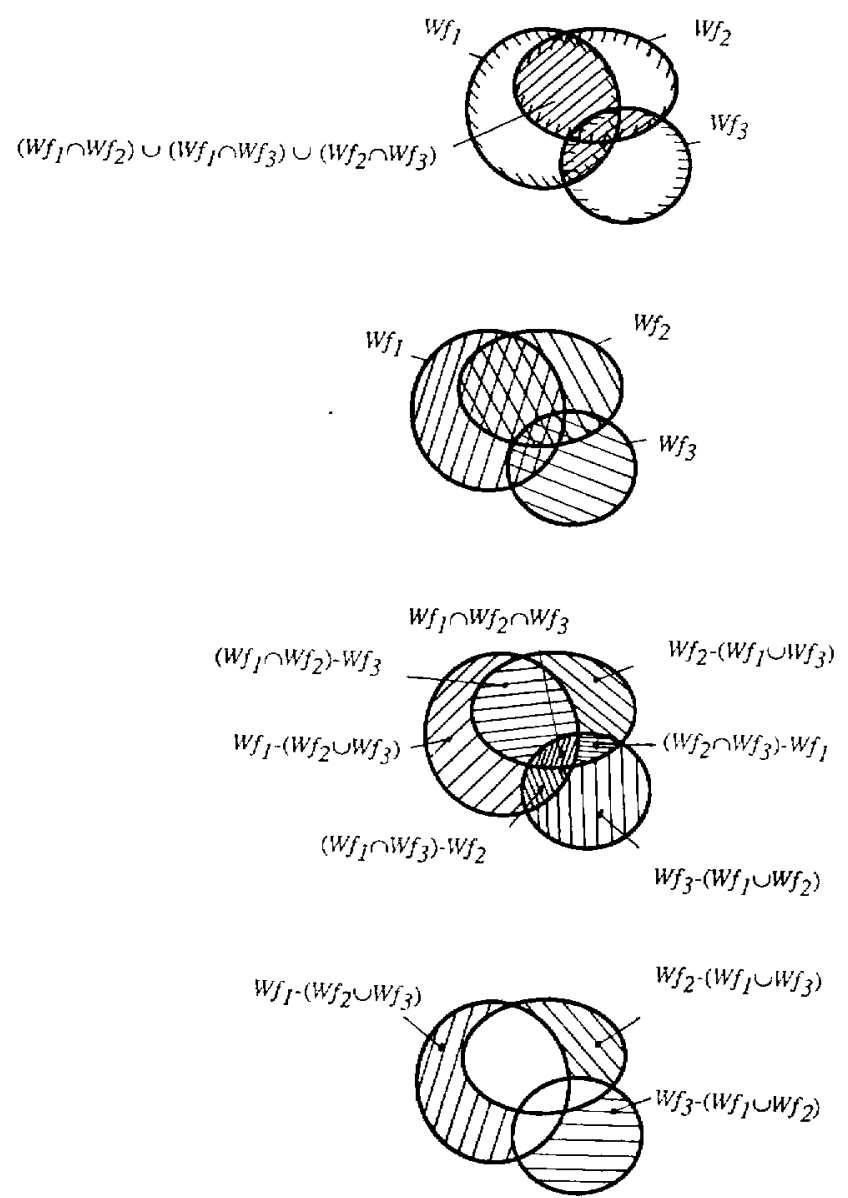

Fig. 4. The different maximal parts of the free work space satisfying (A) $P_{1}$ (Wfp), $P_{2}(\mathrm{Wfp})(\mathrm{B}), P_{3}(\mathrm{Wfp})(\mathrm{C})$ and $P_{4}(\mathrm{Wfp})(\mathrm{D})$ when the configuration collision-free space has three connected components.

Note: A discrete trajectory is an arranged sequence of points.

Wf satisfies $P_{2}$ if and only if:

$$
\exists i \in I, \quad W f_{i}=W f
$$

(See the demonstration in Appendix B.)

Corollary $2 W f \subset W f$ satisfies the generalized property $\mathrm{P}_{2}(W f p)$, where:

$\left(\mathrm{P}_{2}(W f p)\right)$

$\forall \mathbf{T}_{\mathrm{d}}$ discrete trajectory in $W f p, \exists i \in I$ such that $\mathbf{T}_{\mathrm{d}} \subset W f_{i} \cap W f p$

if and only if:

$$
\exists i \in I, \quad W f p \subset W f_{i}
$$

\section{Definition 2}

The maximal parts $W f p$ satisfying $\mathrm{P}_{2}(W f p)$ are defined as follows:

$$
\exists i \in I, \quad W f p=W f_{i}
$$

(see Fig. $4 B$; in the case where $\operatorname{Card}(I)=3$, there are three maximal parts satisfying $\mathrm{P}_{2}(W f p)$ ).

\subsection{Third Characterization and Corresponding Necessary and Sufficient_Condition}

The FW can be traveled through by the robot in the sense of $\mathrm{P}_{\mathbf{3}}$ if, by definition, any two points in $W f$ can be joined by the end effector, whatever the initial or (exclusive or) final configuration:

$\left(\mathrm{P}_{3}\right) \quad \forall(\mathbf{q}, \mathbf{X}) \in Q f \times W f, \exists i \in I, \mathbf{q} \in Q f_{i}$ and $\mathbf{X} \in W f_{i}$

Note: This third property can be expressed in a more succinct way:

$$
\left(\mathrm{P}_{3}\right) \Leftrightarrow W f \times Q f=\cup_{i \in I}\left(W f_{i} \times Q f_{i}\right)
$$

$W f$ satisfies $\mathrm{P}_{3}$ if and only if:

$$
\forall i \in I, \quad W f_{i}=W f
$$

(See the demonstration in Appendix C.)

Corollary 3 Let $W f p \subset W f$ and define:

$$
Q f p=f^{-1}(W f p)
$$

then $W f p$ satisfies the generalized property $\mathrm{P}_{3}(W f p)$, where:

$$
\begin{aligned}
\left(\mathbf{P}_{\mathbf{3}}(W f p) \quad \forall(\mathbf{q}, \mathbf{X}) \in Q f p \times W f p, \exists i \in I, \mathbf{q} \in Q f p \cap Q f_{i}\right. \\
\\
\text { and } \mathbf{X} \in W f p \cap W f_{i}
\end{aligned}
$$

if and only if:

$$
W f p \subset\left(\cap_{i \in I^{\prime}} W f_{i}\right)-\left(\cup_{k \notin I^{\prime}} W f_{k}\right)
$$

for some sets $I^{\prime}$ of indices in $I$. (See the demonstration in Appendix D.)

\section{Definition 3}

The maximal parts $W f p$ satisfying $\mathrm{P}_{3}(W f p)$ are defined as follows:

$$
W f p=\left(\cap_{i \in I^{\prime}} W f_{i}\right)-\left(\cup_{k \notin I^{\prime}} W f_{k}\right)
$$

for any subsets $I^{\prime}$ of $I$ such that $W f p$ is nonempty. (See Fig. $4 C$; when $\operatorname{Card}(I)=3$, there are seven maximal parts satisfying $\mathrm{P}_{3}(W f p)$.) 


\subsection{Fourth Characterization and Corresponding Necessary and Sufficient Condition}

The FW can be traveled through by the robot in the sense of $\mathrm{P}_{4}$ if, by definition, any two points in $W f$ can be joined by the end effector, whatever the initial and final configurations:

$\left(\mathbf{P}_{4}\right) \quad \forall\left(\mathbf{q}_{1}, \mathbf{q}_{2}\right) \in Q f^{2}, \quad \exists i \in I, \mathbf{q}_{1} \in Q f_{i} \quad$ and $\quad \mathbf{q}_{2} \in Q f_{i}$

$W f$ satisfies $\mathrm{P}_{4}$ if and only if $Q f$ is connected (the proof is obvious).

Corollary 4 Let $Q f p$ be defined as in previous corollary 3. $W f p \subset W f$ satisfies the generalized property $\mathrm{P}_{\mathbf{4}}(W f p)$ where:

$\left(\mathbf{P}_{\mathbf{4}}(W f p)\right) \quad \forall\left(\mathbf{q}_{1}, \mathbf{q}_{2}\right) \in Q f p^{2}, \exists i \in I, \mathbf{q}_{1} \in Q f p \cap Q f_{i}$ and $\mathbf{q}_{\mathbf{2}} \in Q f p \cap Q f_{i}$

if and only if:

$$
\exists i \in I / W f p \subset W f_{i}-\left(\cup_{k \neq i} W f_{k}\right)
$$

(See the demonstration in Appendix E.)

\section{Definition 4}

The maximal parts $W f p$ satisfying $\mathrm{P}_{4}(W f p)$ are defined as follows:

$$
\exists i \in I / W f p=W f_{i}-\left(\cup_{k \neq i} W f_{k}\right)
$$

(See Fig. $4 D$; in the case where $\operatorname{Card}(I)=3$, there are three maximal parts satisfying $\mathrm{P}_{4}(W f p)$.)

Note: Properties $\mathrm{P}_{4}$ up to $\mathrm{P}_{1}$ characterize four increasing levels describing point-to-point motions in the FW of a robot:

$$
P_{4} \Rightarrow P_{3} \Rightarrow P_{2} \Rightarrow P_{1}
$$

\subsection{Fifth Characterization and Corresponding Necessary and Sufficient Condition}

This fifth property will concern only nonredundant robots, for which the geometric operator is bijective on the aspects.

The FW can be traveled through by a nonredundant robot in the sense of $\mathrm{P}_{5}$ if, by definition, any two points in $W f$ can be joined with any continuous trajectory $\mathbf{T}_{\mathbf{c}}$ without changing configuration and regardless of the initial or final configuration.

For any $j$ in $J$ (set describing the aspects $A_{j}$ ), define the single partition $\left\{A_{j k}\right\}_{k \in I j}$ of the aspects $A_{j}$ in connected components $A_{j k}$ as:

$$
A_{j} \cap Q f=\cup_{k \in I j} A_{j k}
$$

We note, for $j \in J$ and $k \in I j$ :

$$
W A_{j k}=f\left(A_{j k}\right)
$$

is the image under $f$ of the connected component $k$ of the aspect $j$ in the operational space.

Similarly, we note, for $j \in J$ :

$$
W A_{j}=f\left(A_{j}\right)
$$

is the image of the aspect $j$ in the operational space.

According to the results obtained by Borrel (1986) and generalizing them in the case of environments with obstacles, this fifth property can be expressed as:

$$
\begin{aligned}
& \forall(\mathbf{q}, \mathbf{X}) \in Q f \times W f, \exists j \in J, \exists k \in I_{j} / \mathbf{q} \in A_{j k} \\
& \text { and } \mathbf{X} \in W A_{j k}
\end{aligned}
$$

$W f$ satisfies property $\mathrm{P}_{5}$ if and only if:

$$
\forall j \in J, \forall k \in I_{j} W A_{j k}=W f
$$

The proof is quite analogous to that of theorem 3.

Corollary 5 Let $Q f p$ be defined as in previous corollary 3 . Then $W f p \subset W f$ satisfies the property $\mathrm{P}_{5}(W f p)$, where:

$$
\begin{aligned}
\left(\mathbf{P}_{5}(W f p)\right) & \forall(\mathbf{q}, \mathbf{X}) \in Q f p \times W f p, \exists j \in J, \exists k \in I_{j} / \mathbf{q} \in Q f p \cap A_{j k} \\
& \text { and } \mathbf{X} \in W f p \cap W A_{j k}
\end{aligned}
$$

if and only if:

$$
\begin{aligned}
W f p \subset W A_{j i}-\left(\cup_{j \in J^{\prime}, k \in I^{\prime}(j)} W A_{j k}\right) & \\
& -\left(\cup_{j \in J^{\prime}, l \notin I^{\prime}(j)} W A_{j t^{\prime}}\right)-\left(\cup_{m \notin J^{\prime}} W A_{m}\right)
\end{aligned}
$$

where $J^{\prime}$ is a set of indices in $J$, and, for any $j$ in $J^{\prime}$, $I^{\prime}(j)$ is a set of indices in $I(j)$.

The proof is quite analogous to that of corollary 3 .

\section{Definition 5}

The maximal parts $W f p$ satisfying $\mathrm{P}_{5}(W f p)$ are defined as the connected components of:

$$
W A_{j i}-\left(\cup_{j \in J^{\prime}, k \in I^{\prime}(j)} W A_{j k}\right)-\left(\cup_{j \in J^{\prime}\left(\notin I^{\prime}(j)\right.} W A_{j l^{\prime}}\right)-\left(\cap_{m \notin \mathcal{E}^{\prime}} W A_{m}\right)
$$

\section{Algorithmic Analysis of the Robot's Ability to Travel Through $W f$}

Let $W f p$ be enclosed in (or equal to) $W f$. There exists an infinity of parts $W f p$ that verify $\mathrm{P}_{1}(W f p)$ or $\mathrm{P}_{2}(W f p)$ (any subset of any $W f_{i}$, for instance). The following algorithm tests the properties $\mathrm{P}_{1}, \mathrm{P}_{2}, \mathrm{P}_{3}$, and $\mathrm{P}_{4}$ for $W f$ and leads to all the maximal parts $W f p$ satisfying $\mathrm{P}_{1}(W f p)$ or $\mathrm{P}_{2}(W f p)$.

The robot and its environment are modeled using a CSG CAD technique. It makes it possible to perform collision detection between the robot and its environment (Yu and Khalil 1986; Deligneres 1987). 
1. Determination of $I,\left\{Q f_{i}\right\}_{i \in I},\left\{W f_{i}\right\}_{i \in I}, W f$, Qf: in the case where $n \leqslant 3$ and $m \leqslant 3$, we use an octree (octal tree) description of these spaces in a similar way as in Faverjon (1986). If $n \geqslant 3$, the wrist may be modeled by a circumscribing sphere. The FW and the configuration collisionfree space are obtained by sweeping the configuration space $\mathscr{D}$ according to the joint limits. Collision detection is performed using an efficient algorithm described in Yu (1987). Finally, analysis of the connected components of $Q f$, as described in Samet (1979) and Chedmail and Wenger (1988) leads to $I$ and to the octree definition of $Q f_{i}$ and $W f_{i}$.

2. Verification of the property $\mathrm{P}_{4}$ : If $\operatorname{Card}(I)=1$, then $Q f$ is connected; therefore $\mathrm{P}_{4}$ (and so $\mathrm{P}_{1}, \mathrm{P}_{2}$, $\mathrm{P}_{3}$ ) is true for $W f$; end of the algorithm.

else

3. Verification of the property $\mathrm{P}_{3}$ : If $\forall \mathrm{i} \in \mathrm{I}, W f_{i}=$ $W f$, then $\mathrm{P}_{3}$ is true for $W f$; end of the algorithm. else

4. Verification of the property $\mathrm{P}_{2}$ and determination of the maximal $W f p$ satisfying $\mathrm{P}_{2}(W f p)$ : If $\exists \mathrm{i} \in \mathrm{I}$, $W f_{i}=W f$, the $\mathrm{P}_{2}$ is true for $W f$; the maximal parts satisfying $\mathrm{P}_{2}(W f p)$ are the $W f_{i}$; end of the algorithm.

else

5. Verification of the property $P_{1}$ and determination of the maximal $W f p$ satisfying only $\mathrm{P}_{1}(W f p)$ :

a. Enumerate all possible families of nonempty subsets of $I$. For each such family, say $\left\{I_{k} / k \in K\right\}$, determine

$$
B=\left(\cap_{k \in K}\left(\cup_{j \in I k} W f_{j}\right)\right) \cap\left(\cup_{k \in K}\left(\cap_{j \in I k} W f_{j}\right)\right)
$$

Note: the determination of $B$ is performed using the octree model with very simple and fast boolean operations.

b. If $B$ is nonempty, then it satisfies $\mathrm{P}_{1}(W f p)$; go to $5 \mathrm{a}$.

End of the algorithm.

We can make the following remarks concerning the part 5 of the algorithm:

Note 1: It is possible to considerably reduce the number of cases as a lot of them do not have to be studied:

1. $\operatorname{Card}\left(I_{k}\right)=1$. Then the element $i$ of $I_{k}$ satisfies $W f_{i}=W f p$, already seen
2. There exists $j_{0}$ such that $\forall k \in K, j_{0} \in I_{k}$. Then $W f p \subset W f_{\text {fo }}$ and $W f p$ is not maximal. In particular, if $\operatorname{Card}(K)=1$ : there exists $i$ in $I$ such that $W f p \subset W f_{j}$, and $W f p$ is not maximal.

3. A great number of sequences $\left\{I_{k}\right\}_{k \in K}$ do not have to be tested as they generate nonmaximal parts. For instance, in the case where $N=4$, the sequence $\{(1,2),(2,3),(1,3,4)\}$ does not have to be studied, because the subspace generated by it is enclosed in the maximal $W f p$ generated by the sequence $\{(1,2),(2,3),(1,3)\}$.

Note 2: it is useless to study the case where, in a sequence $\left\{I_{k}\right\}_{k \in K}$, there exists $k$ and $k^{\prime}$ such that $I_{k} \subset$ $I_{k^{\prime}}$. Indeed, let $A_{k}=B$ for any $k \in K$; then

$$
B=\cup_{j \in K^{\prime}}\left(\cap_{i \in I j} W f_{i}\right)=\cup_{j \in K-\left\{k^{\prime}\right\}}\left(\cap_{i \in I j} W f_{i}\right)
$$

and so $W f p$ generated by

$$
\left\{I_{k}\right\}_{k \in K}
$$

is equal to $W f p$, generated by

$$
\left\{I_{k}\right\}_{k \in K-\left\{k^{\prime}\right)}
$$

and do not have to be tested. For example, in the case where $N=3$, it is useless to test the sequence:

$$
\{(1,2),(2,3),(1,3),(1,2,3)\} \text {. }
$$

Therefore we verify that for $N=2$, out of the cases where $W f_{i}=W f$, no subset of $W f$ exists that satisfies $\mathrm{P}_{1}(W f p)$. For $N=3$, then $\mathcal{N}=127$. Out of the cases where $W f_{i}=W f$, one single set of subsets of $I$ may lead to parts of $W f$ that satisfy the condition $\mathrm{P}_{1}(W f p)$ : $\{(1,2),(1,3),(2,3)\}$ where $\operatorname{card}(K)=3$ (see Fig. $4 A$ ).

Similarly, for $N=4$, then $\mathcal{N}=32767$. Out of the cases where $W f_{i}=W f$, we verify that only 27 sets of subsets of $I$ may lead to parts of $W f$ that satisfy the condition $\mathrm{P}_{1}(W f p)$.

\section{Results}

We have developed a package that enables us to study any type of robot in any environment, by only dealing with three-dimensional spaces. In this section, we present some examples, using planar robots for graphic convenience, that illustrate the algorithmic analysis of the moveability in the FW of a robot among obstacles. 


\subsection{Example 1}

The FW is not connected (Fig. 5). In this example, the algorithm detects two connected components in the Cartesian space and concludes that the FW cannot be traveled through in the sense of $P_{1}$ (it cannot enter the box). Two maximal parts $W f_{1}$ and $W f_{2}$ are detected as satisfying $\mathrm{P}_{1}(W f p)$ and $\mathrm{P}_{2}(W f p)$.

\subsection{Example 2}

The FW is connected, and the configuration free space is not connected (Fig. 6). In this example, the algorithm detects two connected components $Q f_{1}$ and $Q f_{2}$ in the configuration space. It verifies that FW cannot be traveled through in the sense of $P_{1}$ (the robot cannot move completely around the spherical obstacle), but the algorithm exhibits two parts $W f_{1}$ and $W f_{2}$ as satisfying $\mathrm{P}_{1}(W f p)$ and $\mathrm{P}_{2}(W f p)$.

\subsection{Example 3}

The FW is connected, and the configuration free space is not connected (Fig. 7). In this case, the algorithm detects two connected components $Q f_{1}$ and $Q f_{2}$ in the configuration free space and exhibits two parts $W f_{1}$ and $W f_{2}$ such that $W f_{2} \subset W f$ and $W f_{1}=W f$. So the algorithm concludes that the FW can be traveled through in the sense of $\mathrm{P}_{2}(W f p)$ (and $\mathrm{P}_{1}(W f p)$ ).

We verify that $W f_{2}$ can be traveled through in the sense of $\mathrm{P}_{3}(W f p)$ and the subspace $W f-W f_{2}$ in the sense of $\mathrm{P}_{4}(W f p)$.

\subsection{Example 4}

The FW and the configuration collision-free space are both connected (Fig. 8). In this example, the algorithm finds only one single connected component in the sense of $\mathrm{P}_{4}$ (not in the sense of $\mathrm{P}_{5}$, as we could verify that the configuration space is composed of two aspects, whose images in the operational space are different).

\subsection{Comments}

As it has been shown in these examples, a simple connectivity analysis of the configuration collision-free space is not sufficient to characterize the geometric (a)

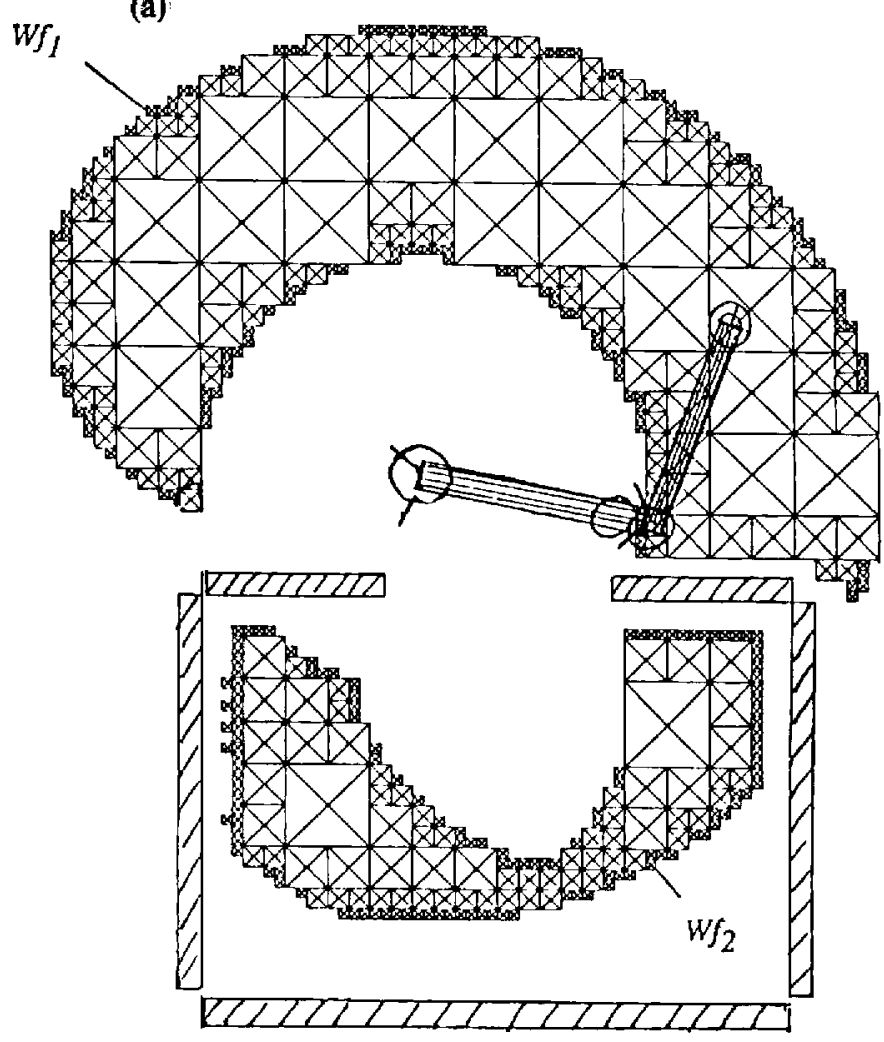

Fig. 5. The free work space is not connected (A, Cartesian space); the configuration collision-free space is composed of two connected components $Q f_{1}$ and $Q f_{2}(\mathrm{~B}$, configuration (b)
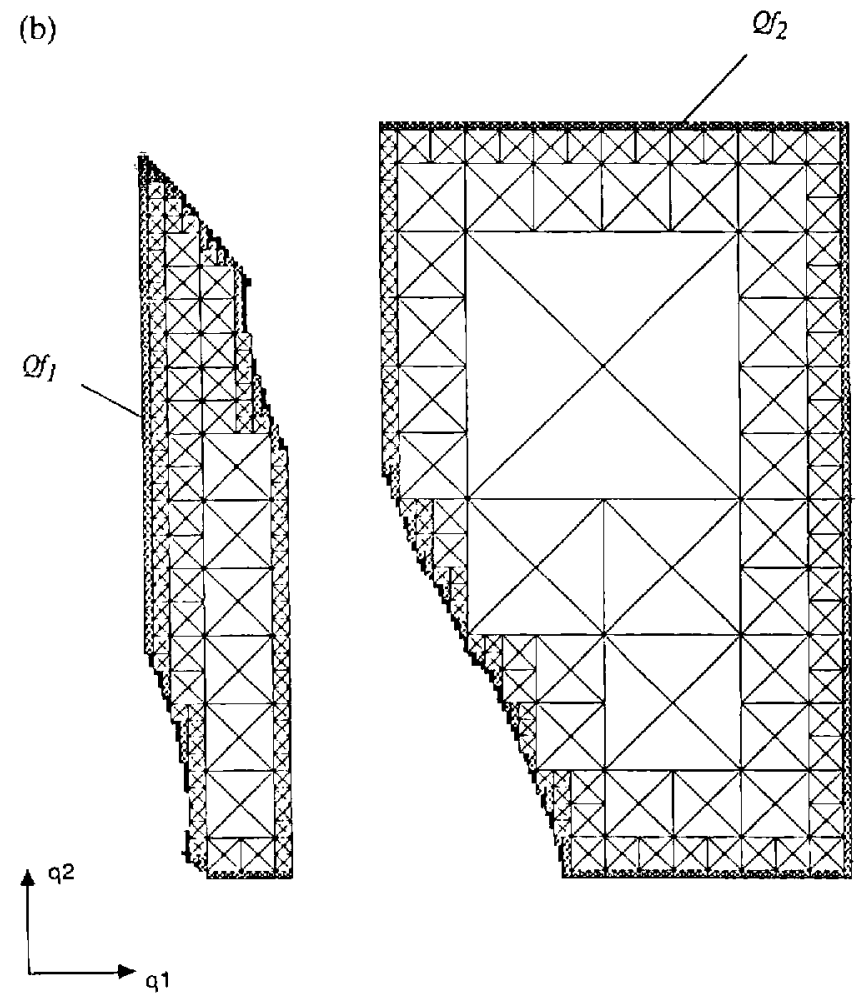

space), and their image in the operational space (Wf and $\left.W f_{2}\right)$ satisfies property $P_{1}(\mathrm{Wfp})$. 

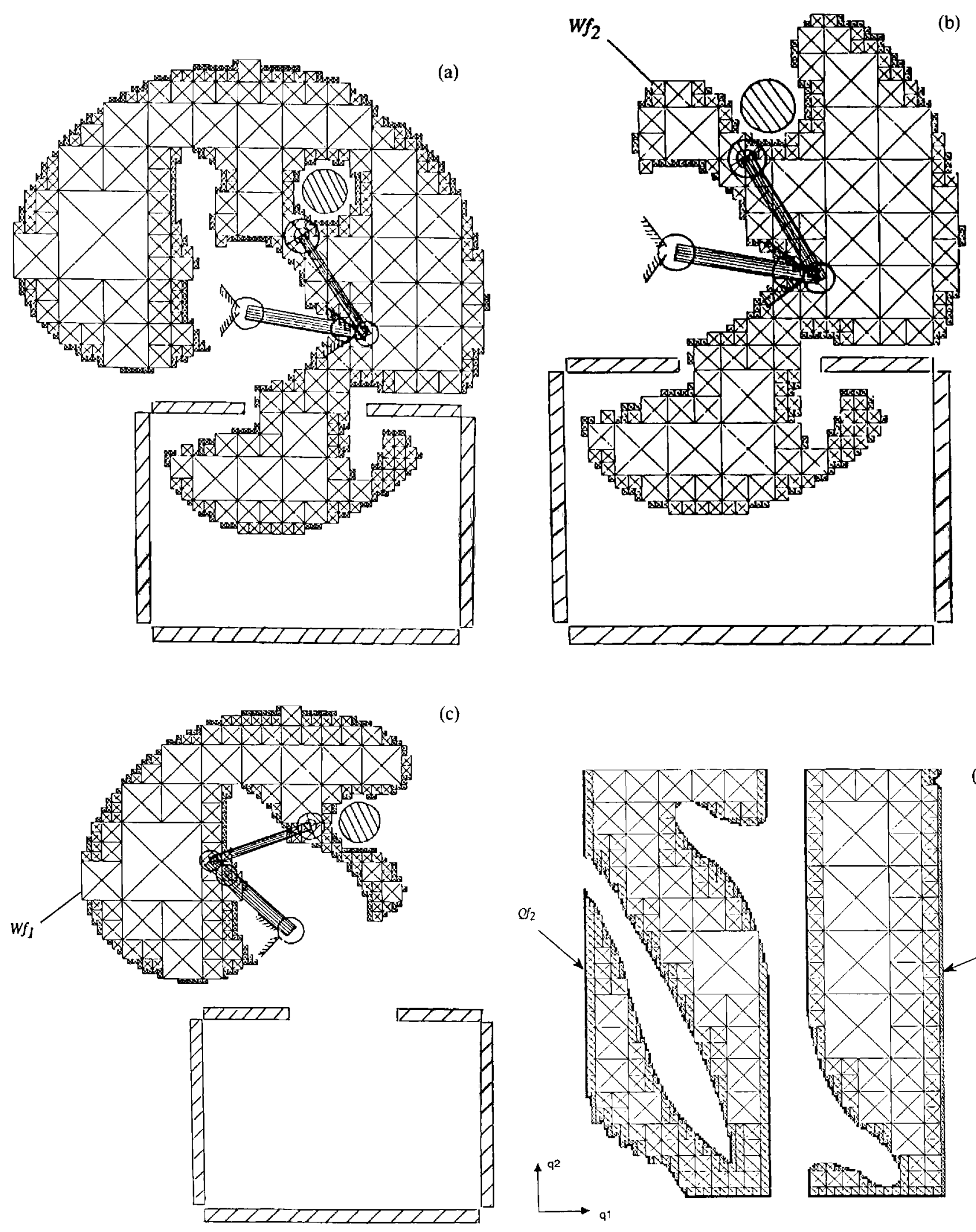

Fig. 6. The free work space is connected (A, Cartesian space); the configuration collision-free space is composed of two connected components $Q f_{1}$ and $Q f_{2}(\mathrm{D}$, configuration space)

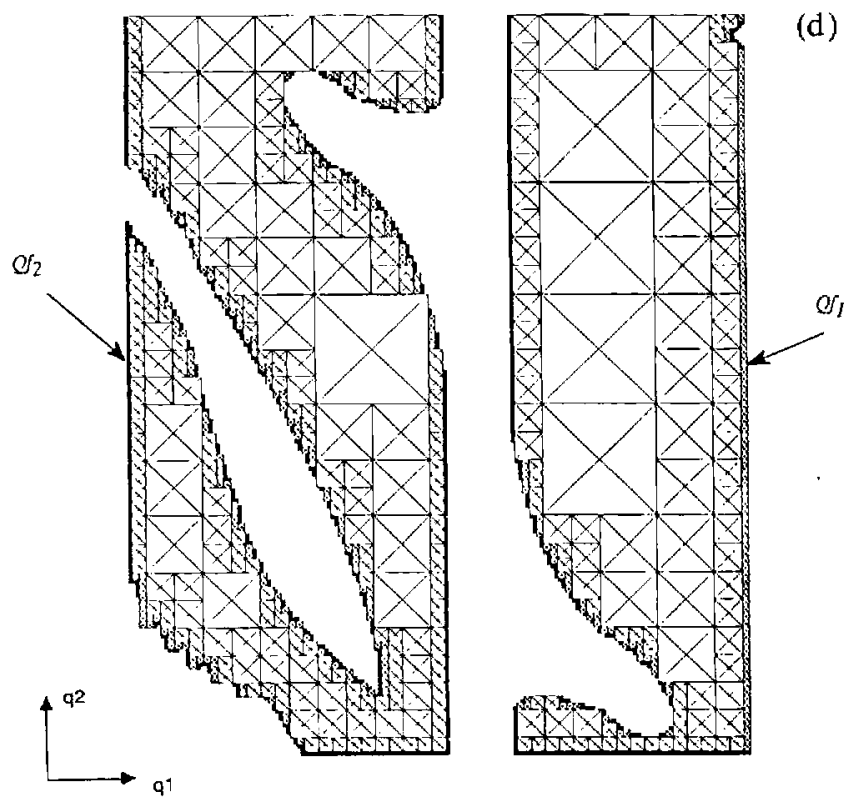

and their image in the operation space $\left(W f_{1}\right.$ and $\left.W f_{2}\right)$ satisfies property $P_{l}(\mathrm{Wfp})(\mathrm{B}$ and $\mathrm{C})$. 

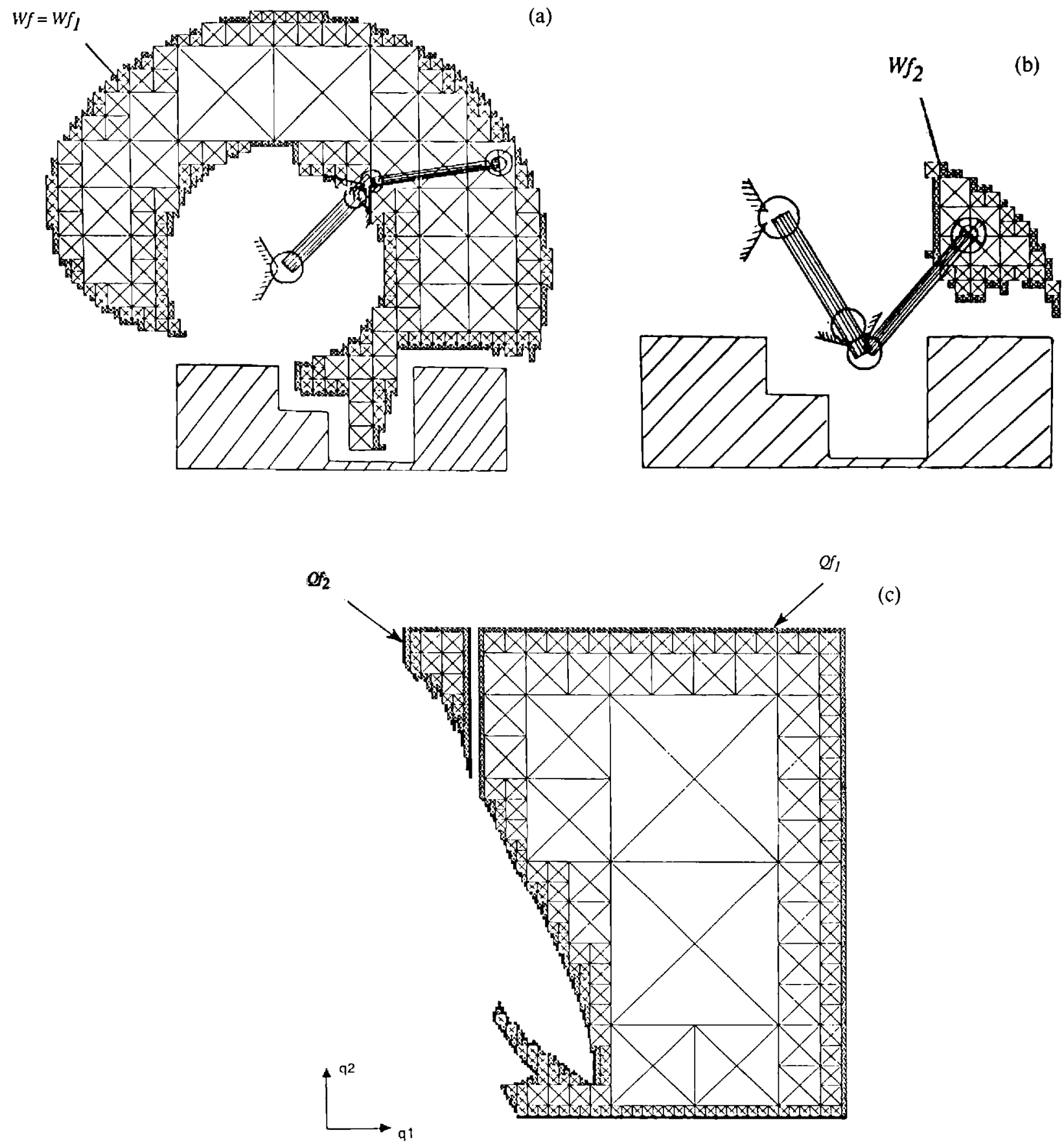

(c)

Fig. 7. The robot is able to travel through its free work space in the sense of $P_{2}$ (A, Cartesian space) and through the subspace $W f_{2}$ in the sense of $P_{3}(\mathrm{Wfp})(\mathrm{B})$. The configuration

properties of the free work space. The configuration collision-free space may be nonconnected, whereas the FW can be traveled through (in the sense of $P_{1}, P_{2}$, or $P_{3}$; see example 3). On the other hand, the configuration collision-free space may be connected, whereas collision-free space is composed of two connected components (C, configuration space).

the FW cannot be traveled through (in the sense of $P_{5}$; see example 4).

The analysis of this paradox and the characterizations of the moveability of the robot in its FW are the main contributions of the present work. 

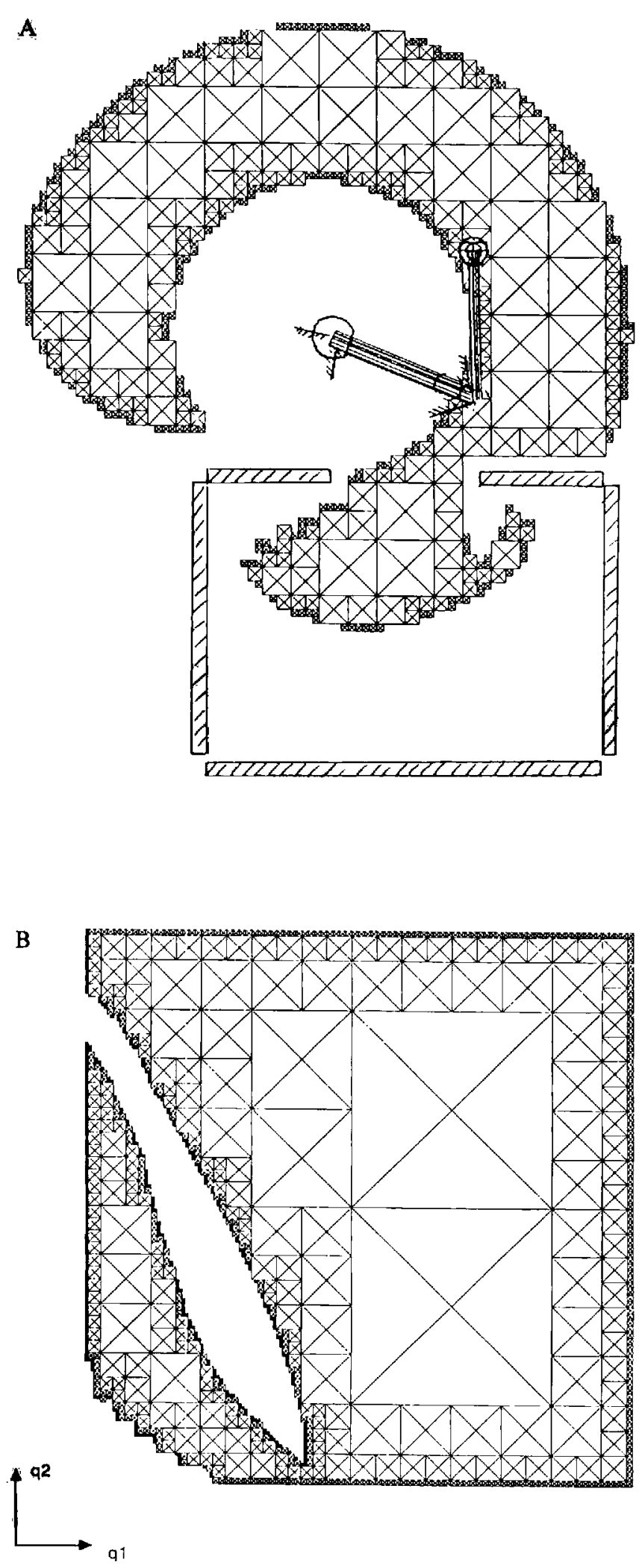

Fig. 8. The robot is able to travel through its free work space in the sense of $\mathrm{P}_{4}(\mathrm{~A}$, Cartesian space) as the configuration free space is connected ( $\mathrm{B}$, configuration space).

\section{Conclusion}

This article presents a classification, in the operational space, of regions where the robot is able to achieve motions according to its joint limits and the obstacles lying in the environment. Five characterizations of the moveability in the work space have been explained, as well as the five corresponding necessary and sufficient conditions. Based on these conditions, an algorithm has been developed that tests whether $W f$ satisfies $\mathrm{P}_{1}$ or $\mathrm{P}_{2}$ or $\mathrm{P}_{3}$ or $\mathrm{P}_{4}$ and finds all the parts of $W f$ satisfying $\mathrm{P}_{1}(W f p)$ or $\mathrm{P}_{2}(W f p)$. Any robot in any environment can be studied, provided that the dimensions of the spaces are not greater than three (our algorithm is quite general and could theoretically deal with $k$ dimensional trees, with $k>3$, but this would lead to prohibitive computational time; when $k \leqslant 3$, the number of tests is reasonable and compatible with the capacity of current minicomputers).

This work is a new contribution to the design of robotic cells. The geometric performances of the robot among the various obstacles of the cell can be evaluated through the five characterizations of its FW. This is an interesting aid for the morphology choice of a robot. In addition, the geometric layout of the cell may be realized by determining the suitable moveability areas in the environment. Independently, we have developed a methodology that performs the automatic positioning of a robot in its environment in order to reach a given area (Chedmail and Wenger 1989). This methodology and the present study are currently integrated in a software package that defines the automatic positioning of a robot among the various obstacles.

\section{Appendix A. Demonstration of Property $\mathbf{P}_{\mathbf{1}}$}

\section{Sufficient Condition}

Let $\mathbf{x}_{1} \in W f, \mathbf{x}_{2} \in W f$ :

$$
\exists k \in K / \mathbf{X}_{1} \in \cap_{j \in I k} W f_{j} \text { (from } i i \text { ), }
$$

and so

$$
\exists j \in I_{k} / \mathbf{X}_{1} \in W f_{j}, \text { and } \mathbf{X}_{2} \in W f_{j} \text { (from } i \text { ), }
$$

therefore:

$$
\forall \mathbf{X}_{1}, \mathbf{X}_{2} \in W f, \exists j \in I, \mathbf{X}_{1} \in W f_{j} \text { and } \mathbf{X}_{2} \in W f_{j} \text {, }
$$

That is the property $P_{1}$.

\section{Necessary Condition}

Suppose $W f$ is not connected, then no trajectory exists between the different connected components of it, and so it does not satisfy the property $P_{1}$. 
So we suppose that $W f$ is connected and satisfies the property $P_{1}$ :

$\left(\mathrm{P}_{1}\right): \quad \forall \mathbf{X}_{1}, \mathbf{X}_{2} \in W f, \exists j \in I, \mathbf{X}_{1} \in W f_{j} \quad$ and $\quad \mathbf{X}_{2} \in W f_{j}$.

Let

$$
I\left(\mathbf{X}_{1}\right)=\left\{j \in I / \mathbf{X}_{1} \in W f_{j}\right\}
$$

Hence

$$
\begin{aligned}
W f=\cup_{j \in I(X 1)} W f_{j} \\
\text { and } \\
\mathbf{X}_{1} \in \cap_{j \in I(X 1)} W f_{j}
\end{aligned}
$$

as $P_{1}$ is satisfied.

Therefore

$$
\forall \mathbf{X}_{1} \in W f, \exists I\left(\mathbf{X}_{1}\right) \subset I
$$

such that

$$
W f=\cup_{j \in I(x))} W f_{j}
$$

and

$$
\mathbf{X}_{1} \in \cap_{j \in I(X 1)} W f_{j}
$$

Elsewhere

$$
\exists K /\left\{I_{k}\right\}_{k \in K}=\left\{I\left(\mathbf{X}_{1}\right)\right\}_{X 1 \in W f} .
$$

$K$ is obtained by sweeping the finite set of the parts of $I$, the cardinal of which is finite: $\operatorname{Card}(\mathbf{K}) \leqslant 2^{\mathrm{N}}-1$.

Finally

$$
\forall k \in K, W f=\cup_{j \in I k} W f_{j}
$$

from (1) and (3), and

$$
W f \subset\left(\cap_{j \in I k} W f_{j}\right)
$$

from (2) and (3); therefore $i$ and $i i$ are satisfied.

\section{Appendix B. Demonstration of Property $\mathbf{P}_{\mathbf{2}}$}

\section{Sufficient Condition}

It is obvious.

\section{Necessary Condition}

Suppose that: $\forall i \in I, W f_{i} \subset W f$ (strictly). Thus, for any $i$ in $I, D f_{i}=W f_{i}-W f$ is nonempty.

Let $\mathbf{T}_{\mathrm{d}}=\left\{\mathbf{X}_{1}, \mathbf{X}_{2}, \ldots, \mathbf{X}_{\mathbf{p}}\right\}$ be a discrete trajectory in $W f$, where $p$ is the number of connected components of the configuration collision-free space: $p=$ $\operatorname{Card}(I)$. This trajectory is chosen such that for any $j \leqslant$ $p, \mathbf{X}_{\mathbf{j}}$ is in $D f_{j}$. Thus there exists a discrete trajectory in $W f$ such that $\forall i \in I, \mathbf{T}_{\mathrm{d}} \cap D f_{i} \neq \varnothing$, which means:

$$
\exists \mathbf{T}_{\mathrm{d}} \subset W f / \forall i \in I, \mathbf{T}_{\mathbf{d}} \not \subset W f \text {. }
$$

\section{Appendix C. Demonstration of Property $\mathbf{P}_{\mathbf{3}}$}

Sufficient Condition

Let $\mathbf{X}_{1} \in W f$ and $\mathbf{q}_{1} \in Q f$ such that $\mathbf{X}_{1}=f\left(\mathbf{q}_{1}\right)$.

$$
\forall i \in I, \mathbf{X}_{1} \in W f_{i} \text { and } \mathbf{q}_{1} \in Q f_{i} \text {. }
$$

Similarly, let $\mathbf{X}_{2} \in W f$, then $\forall i \in I, \mathbf{X}_{2} \in W f_{i}$, and so $\mathrm{P}_{3}$ is satisfied.

\section{Necessary Condition}

Suppose $\exists i \in I$ such that $W f_{i} \subset W f$ (strictly); let $\mathbf{q}_{\mathbf{1}} \in$ $Q f_{i}$ and let $\mathbf{X}_{2} \in W f-W f_{i}$; then $\mathbf{P}_{3}$ is false.

\section{Appendix D. Demonstration of Corollary 3 (Property $\mathrm{P}_{\mathbf{3}}(\boldsymbol{W f p})$ )}

\section{Sufficient Condition}

Let $W f p \subset\left(\cap_{i \in I^{\prime}} W f_{i}\right)-\left(\cup_{k \notin I^{\prime}} W f_{k}\right)$ for some $I^{\prime}$ in $I$.

Let $\mathbf{q}_{1}$ in $Q f p$ and let $\mathbf{X}_{1}=f\left(\mathbf{q}_{1}\right)$.

As $\mathbf{X}_{1} \notin W f_{k}$ for any $k$ not belonging to $I^{\prime}$, there exists $i_{0}$ in $I^{\prime}$ such that $\mathbf{q}_{1} \in Q f p \cap Q f_{i 0}$.

Let $\mathbf{X}_{2}$ in $W f p: \forall i \in I^{\prime}, \mathbf{X}_{2} \in W f p \cap W f_{i}$ and thus $\mathbf{X}_{2} \in W f p \cap W f_{i 0}$.

\section{Necessary Condition}

Let $D\left(I^{\prime}\right)=\left(\cap_{i \in I^{\prime}} W f_{i}\right)-\left(\cup_{k \notin I^{\prime}} W f_{k}\right)$. The sets $D\left(I^{\prime}\right)$ make a partition of $W f$, when considering all possible subsets $I^{\prime}$ in $I$. Indeed, they are clearly all disjointed, and moreover:

$$
\cup_{I^{\prime} \subset I} D\left(I^{\prime}\right) \supset \cup_{i \in I^{\prime}} W f_{i}-\cup_{i \in I}\left(\cap_{k \notin I} W f_{k}\right)=W f .
$$

Now, let $W f p$ in $W f$, and suppose $\forall I^{\prime} \subset I, W f p \not \subset$ $D\left(I^{\prime}\right)$. Then, there exists $I^{\prime}$, and $I^{\prime}$ in $I$ such that $W f p \cap$ $D\left(I^{\prime}{ }_{1}\right) \neq \varnothing$ and $W f p \cap D\left(I^{\prime}{ }_{2}\right) \neq \varnothing$. If $I^{\prime}{ }_{1} \not \subset I^{\prime}{ }_{2}$, then there exists $\mathbf{q}_{1}$ in $f^{-1}\left(W f p \cap D\left(I^{\prime}{ }_{1}\right)\right)$ such that $\mathbf{q}_{1}$ belongs to $Q f_{i 0}$ for $i_{0}$ in $I^{\prime}{ }_{1}-I^{\prime}{ }_{2}$. Let $\mathbf{X}_{2}$ in $W f p \cap D\left(I^{\prime}{ }_{2}\right)$; then the property $\mathrm{P}_{3}(W f p)$ is false. Similarly, if $I_{1} \subset$ $I^{\prime}{ }_{2}$, there exists $\mathbf{q}_{1}$ in $f^{-1}\left(W f p \cap D\left(I^{\prime}{ }_{1}\right)\right)$ such that $\mathbf{q}_{1}$ belongs to $Q f_{i 0}$ for $i_{0}$ in $I_{2}^{\prime}-I_{2}$. Let $\mathbf{X}_{2}$ in $W f p \cap$ $D\left(I_{2}^{\prime}\right)$; then the property $\mathrm{P}_{3}(W f p)$ is false.

\section{Appendix E. Demonstration of Corollary 4 (Property $\mathrm{P}_{\mathbf{4}}(\boldsymbol{W f p})$ )}

\section{Sufficient Condition}

Let $\left(\mathbf{X}_{1}, \mathbf{X}_{2}\right) \in W f p \times W f p$. Then

$$
\exists i \in I /\left(\mathbf{X}_{1}, \mathbf{X}_{2}\right) \in\left(W f_{i} \cap W f p\right) \times\left(W f_{i} \cap W f p\right),
$$


and

$$
\left(\mathbf{X}_{1}, \mathbf{X}_{2}\right) \notin\left(\cup_{k \neq i} W f_{k} \cap W f p\right) \times\left(\cup_{k \neq i} W f_{k} \cap W f p\right),
$$

which proves that $\exists i \in I / \mathbf{q}_{1} \in Q f p \cap Q f_{i}$ and $\mathbf{q}_{2} \in$ $Q f p \cap Q f_{i}$.

\section{Necessary Condition}

Suppose $\forall i \in I, W f p \not \subset W f_{i}-\left(\cup_{k \neq i} W f_{k}\right)$. Let $\mathbf{X}_{1} \in$ $W f_{i}-\left(\cup_{k \neq i} W f_{k}\right)$; then $\mathbf{q}_{1} \in Q f_{i}$. Let $\mathbf{X}_{2} \in W f p-$ $\left(W f_{i}-\left(\cup_{k+i} W f_{k}\right)\right)$; then $\mathbf{q}_{2} \notin Q f_{i}$, which proves that $W f p$ does not satisfy $\mathrm{P}_{4}(W f p)$.

\section{Acknowledgment}

This work has been partially supported by the MRES contract MEC/10/10: "Choix d'architectures de robots" (Robots morphology choice).

\section{References}

Bernard, F. 1984. CATIA: du dessin au volume, de la cinématique aux calculs scientifiques, de la commande numérique à la robotique, un outil complet de C.F.A.O. Proc. M.I.C.A.D., (2):1082- 1097.

Borrel, P. 1986. A study of multiple manipulator inverse kinematic solutions with applications to trajectory planning and workspaces determination. IEEE Conf. Robot. Automat., pp. 1181-1185.

Brady, M., Hollerbach, J. M., Johnson, T. L., et al. 1982. Robot Motion: Planning and Control. Cambridge, Mass.: MIT Press.

Brooks, R. A. 1983a. Planning collision free motions for pick-and-place operations. Int. J. Robot. Res. 2(4):19-44.

Brooks, R. A. 1983 b. Solving the path-finding problem by good representation of free space. IEEE Trans. Sys. Man. Cybernet. SMC-13:190-197.

Canny, J. F. 1987 (Los Angeles). A new algebraic method for robot motion planning and real geometry. Presented at IEEE 28th symposium.

Canny, J. F., and Reif, J. 1987 (Los Angeles). New lower bound techniques for robot motion planning problems. Presented at IEEE 28th symposium.

Canny, J. F., and Donald, B. R. 1988. Simplified Voronoi diagrams. Discrete Computat. Geomet. 3(3):219-236.

Chedmail, P., and Wenger, P. 1988 (Karlsruhe, F.R.G.). Ability of a robot to move between two points within cartesian free workspace with an encumbered environment. IFAC Symposium on Robots Control, pp. 73.1-73.6.

Chedmail, P., and Wenger, P. 1987 (Nantes, France). Domaine atteignable par un robot: Généralisation de la notion d'aspects a un environnement avec obstacles. Application aux robots articulés plans. Proc. AUM Symposium $(2): 312-313$
Chedmail, P., and Wenger, P. 1989 (Scottsdale, Arizona, May 14-19). Design and positioning of a robot in an environment with obstacles using optimal research. IEEE Conf. Robot. Automat. Vol. 2.

Deligneres, S. 1987. Choix de morphologies de robots. Ph.D. thesis, E.N.S.M., Nantes, France.

Dombre, E., Fournier, A., Quaro, C., and Borrel, P. 1986. Trends in CAD/CAM systems for robotics. IEEE Conf. Robot. Automat., pp. (3):1913-1918.

Faverjon, B. 1984 (Atlanta). Obstacle avoidance using an octree in the configuration space of a manipulator. 1 st IEEE Conf. Robot. Automat., pp. (1):504-512.

Faverjon, B. 1986. Object level programming of industrial robots. IEEE Int. Conf. Robot. Automat. 3:1406-1412.

Gupta, K. C., and Roth, B. 1982. Design considerations for manipulators workspace. ASME J. Mechan. Des. 104:704711.

Hansen, J. A., Gupta, K. C. and Kazerounian, S. M. K. 1983. Generation and evaluation of the workspace of a manipulator. Int. J. Robot. Res. 2(3);22-31.

Khalil, W., and Kleinfinger, J. F. 1986. A new geometric notation for open and closed-loop robots. IEEE Int. Conf. Robot. Automat. 2:1174-1179.

Khatib, O. 1985 (St. Louis). A real time obstacle avoidance for manipulator and mobile robots. IEEE Conf. Robot. Automat. pp. 500-505.

Koditschek, D. 1987 (Raleigh). Exact robot navigation by means of potential functions: Some topological considerations. Proc. IEEE Int. Conf. Robot. (1):1-6.

Kumar, A., and Waldron, K. 1981. The workspaces of mechanical manipulators. ASME J. Mechan. Des. 103:665672.

Lenarcic, J., Stanic, U., and Oblak, P. 1988 (Ljubljana, Yugoslavia). Optimum design of robot mechanisms based on workspace analysis. Proc. Adv. in Rob. Kin., (1):59-165.

Lozano-Pérez, T. 1986 (Philadelphia). A simple motion planning algorithm for general robot manipulators. Proc. Fifth Nat. Conf. for the Am. Assoc. of Art. Int., pp. 626-631.

Lozano-Pérez, T, et al. 1987. Handley: A robot system that recognises, planes and manipulates. Proc. IEEE Int. Conf. Robot. Automat., (2):843-849.

Martin, D., Martin, P., Plemenos, D. 1985. La modèlisation des solides. Nantes, France, Université de Nantes, Institut de mathématiques et d'informatique. Res. rep. IMI-INFOR20.

O'Dunlaing, C., Sharir, M., and Yap, C. 1984. Generalized Voronoi diagrams for moving a ladder. NYU-Courant Institute, Robotics Lab., Technical rep. no. 32-33.

Paden, B., and Sastry, S. 1988. Optimal kinematics design of 6R manipulators. Int. J. Robot. Res. 7(2):43-61.

Samet, H. 1979. Connected component labeling using quadtrees. College Park, Maryland, Computer Science Department, University of Maryland.

Schwartz, J., and Sharir, M. 1982. On the piano mover's problem: General techniques for computing topological properties of real algebraic manifolds. New York, N.Y., Computer Science Department, New York University, report no. 41 . 
Vijiaykumar, R., Waldron, J., and Tsai, M. J. 1986. Geometric optimization of serial chain manipulator structures for working volume and dexterity. Int. J. Robot. Res. 5(2):91-103.

Wenger, P. 1985. Modélisation du robot et de l'environnement en vue de la détection de collision. Nantes, France, D.E.A., Université de Nantes, E.N.S.M.
Yang, D. C. H., and Chiueh, T. S. 1986. Workspace of 6joints robots with fixed hand orientation. Int. J. Robot. Automat. 1(1):23-32.

Yu, Z. Q., and Khalil, W. 1986 (Vienna, Dec. 3-5). Table look up for collision detection and safe operation of robots. IFAC Symposium on Theory of Robots, pp. 407477. 